\title{
Effets comparatifs de la préparation mécanique du sol et de l'application répétée d'un phytocide chimique pour maîtriser le nerprun et favoriser la croissance en plantations forestières
}

\author{
par Sébastien Debar ${ }^{1}$, François Hébert ${ }^{2}$, Martin Barrette ${ }^{3}$, Maxime Brousseau² et Nelson Thiffault²,3,4
}

\begin{abstract}
RÉSUMÉ
Le nerprun bourdaine (Frangula alnus) est une espèce exotique envahissante qui compromet la croissance de plantations dépinette blanche (Picea glauca) au Québec. L'application de phytocides chimiques, le plus souvent de manière répétée, est la seule approche reconnue pour maîtriser le nerprun. Toutefois, son utilisation soulève des enjeux sociaux et environnementaux. Ainsi, notre objectif était dévaluer les effets de la préparation mécanique du sol comme outil de maîtrise du nerprun sur des sites reboisés. Nous avons comparé, après quatre ans, trois traitements mécaniques du sol (incluant la mise en andains, à titre de témoin) et un scénario d’applications répétées d'un phytocide chimique dans un dispositif expérimental en blocs complets. Globalement, la préparation mécanique du sol par hersage ou broyage n’a pas eu deffet à court terme sur la maîtrise du nerprun et sur les dimensions de lépinette blanche par rapport à la mise en andains. La mise en andains suivie de l'application répétée d'un phytocide chimique a eu un effet significatif, bien que limité, sur la hauteur modale du nerprun. Le traitement chimique na par ailleurs pas stimulé la croissance en hauteur des épinettes plantées, mais a significativement augmenté leur diamètre. La préparation mécanique du sol par hersage ou broyage ne semble pas pouvoir remplacer l'application répétée de phytocides chimiques après une mise en andains pour maitriser le nerprun. L’envahissement par le nerprun soulève donc un enjeu majeur de maintien de la productivité des écosystèmes plantés, particulièrement dans un contexte où les phytocides chimiques ne peuvent être utilisés. Les changements globaux risquent d'exacerber cet enjeu, de même que celui associé à l’envahissement des écosystèmes naturels en facilitant lexpansion du nerprun dans de nouvelles aires de distribution.
\end{abstract}

Mots-clés : changements globaux, espèce exotique envahissante, Frangula alnus, Rhamnus frangula, nouveaux écosystèmes, sylviculture, gestion de la végétation

\section{ABSTRACT}

Glossy buckthorn (Frangula alnus) is an exotic, invasive species that reduces white spruce (Picea glauca) plantation growth in Québec. Chemical herbicide applications, usually repeated more than once, are the only vegetation management approach known to control the species effectively. Its use, however, raises social and environmental issues. Our objective was thus to evaluate mechanical site preparation as a vegetation management tool to control glossy buckthorn on reforestation sites. We compared, after four years, three mechanical site preparation treatments (including windrowing as a control) and a scenario of repeated chemical herbicide applications in a fully randomized complete block design. Globally, mechanical site preparation by harrowing or mixing did not have significant short-term effect on buckthorn control and planted spruce dimensions, compared to windrowing. Windrowing followed by repeated chemical herbicide applications had a significant but limited effect on buckthorn modal height. Whereas the chemical treatment did not promote height growth of planted seedlings, it significantly increased diameter. Hence, mechanical site preparation using harrowing or mixing does not seem to be an effective alternative to repeated chemical herbicide applications following windrowing to control glossy buckthorn. Site invasion by this species thus raises a major issue regarding planted ecosystems productivity, especially in contexts where chemical herbicides cannot be used. Global changes might exacerbate this issue as well as the invasion of natural forests by extending the geographic distribution of the species.

Keywords: global changes, exotic invasive species, Frangula alnus, Rhamnus frangula, new ecosystems, silviculture, vegetation management

\footnotetext{
${ }^{1}$ Faculté de foresterie, de géographie et de géomatique, Université Laval, 2405, rue de la Terrasse, Québec (Québec) G1V 0A6

${ }^{2}$ Centre détude de la forêt, Université Laval, 2405, rue de la Terrasse, Québec (Québec) G1V 0A6

${ }^{3}$ Direction de la recherche forestière, Ministère des Forêts, de la Faune et des Parcs, 2700, rue Einstein, Québec (Québec) G1P 3W8

${ }^{4}$ Auteur de correspondance. Adresse actuelle : Centre canadien sur la fibre de bois, Service canadien des forêts, Ressources naturelles

Canada, 1055, rue du P.E.P.S., C.P. 10380, succ. Sainte-Foy, Québec (Québec) G1V 4C7 (nelson.thiffault@canada.ca)
} 


\section{Introduction}

L'envahissement des écosystèmes naturels par des espèces exotiques est un enjeu écologique et économique important de l'anthropocène (Mack et al. 2000; Pimentel et al. 2002; Hulme 2009; Morse et al. 2014; Lennon 2015). Les changements globaux et la globalisation qui caractérisent cette période ont permis à plusieurs espèces détablir de nouvelles aires de distributions où elles sont considérées comme des espèces exotiques (Morse et al. 2014; Lennon 2015). On considère qu'une espèce exotique est envahissante lorsqu'elle établit une nouvelle aire de distribution dans laquelle elle prolifère, sétend et persiste au détriment de lécosystème naturel (Mack et al. 2000). Une espèce exotique peut devenir envahissante, entre autres, si elle peut se reproduire, sétablir et croitre agressivement en absence de maladies ou d'herbivores pouvant limiter son expansion (Gassmann et al. 2008). Les plantes exotiques envahissantes ont des effets néfastes sur la biodiversité et le fonctionnement des écosystèmes naturels (Vilà et al. 2011; Krumm et Vítková 2016). Ceci se répercute sur l'économie en raison des pertes de services écosystémiques et de la mise en place de méthodes de maîtrise et de suivi (U.S. Congress 1993; Pimentel et al. 2002). Au Canada, les coûts annuels engendrés par les espèces exotiques envahissantes sont de l'ordre de 20 G\$ pour le secteur forestier (Environnement Canada 2012).

Le nerprun bourdaine (Frangula alnus Mill., syn. de Rhamnus frangula L.) qui est naturellement présent en Europe (Medan 1994), dans le nord de l'Afrique et en Asie (Converse 1984), est une espèce exotique envahissante en Amérique du Nord. Introduite aux États-Unis au début des années 1800 (Howell et Blackwell 1977), cette espèce est maintenant répandue dans le nord-est de l'Amérique du Nord où elle entrave le recrutement et la croissance des espèces forestières indigènes (Fagan et Peart 2004; Hamelin et al. 2016; Hamelin et al. 2017). Lespèce a la capacité d’occuper un large éventail de types de sols et de milieux, tels que les zones humides non inondées (Catling et Porebski 1994), les rives (Krock et Williams 2002), les plaines ou les sols sablonneux (Gassmann et al. 2008; Lee et Thompson 2012). Le nerprun ne tolère toutefois pas les longues périodes d'inondation (Fiedler et Landis 2012). Cette espèce exotique est envahissante en raison de sa production abondante de graines tôt dans son développement, de même qu'en raison de son établissement et de sa croissance rapide (Gassmann et al. 2008; Hamelin et al. 2015). De plus, sa plasticité architecturale lui permet d'optimiser la captation de la lumière disponible en adoptant une forme arborescente en sous-étage et une forme arbustive dans les ouvertures (Hamelin et al. 2015). Enfin, le nerprun bourdaine peut offrir une compétition pour la lumière avec les espèces indigènes jusquà une hauteur de sept mètres (Gassmann et al. 2008). Cette compétition est exacerbée par le fait que les feuilles de cette espèce apparaissent tôt au printemps et tombent tard en automne (Hamelin et al. 2015).

Le nerprun bourdaine est abondant dans l'est du Canada; on le trouve notamment au Québec, au Nouveau-Brunswick et en Nouvelle-Écosse (Haber 2002). Au Québec, l'espèce est surtout observée dans le domaine bioclimatique de l'érablière à tilleul (Saucier et al. 2009). En plus dêtre présent en peuplement naturel, elle envahit des plantations forestières, notamment celles d'épinette blanche (Picea glauca (Moench) Voss) du sud-ouest du Québec, où elle compromet l'établissement et la croissance des plants mis en terre. Le dégagement mécanisé réalisé à l'aide de débroussailleuses s'est révélé inefficace pour gérer cette espèce en raison de l'importante production de rejets de souches (Boulet et al. 2013). Larrachage manuel est efficace, mais peu envisageable sur de grandes superficies ou bien lorsque la densité est élevée (Hackett et al. 2014). À ce jour, l'application de phytocides chimiques, le plus souvent de manière répétée, est la seule approche reconnue pour contrôler cette espèce exotique envahissante (Nagel et al. 2008; Au et Tuchscherer 2014). Toutefois, l'application de phytocides chimiques soulève des enjeux sociaux et écologiques, si bien qu'elle n'est plus permise dans les forêts publiques de certaines juridictions, dont celles du Québec (Thiffault et Roy 2011).

Par ailleurs, la préparation mécanique des sols peut limiter la compétition de certaines espèces concurrentes pendant les premières années suivant la mise en terre des plants (Wiensczyk et al. 2011). Laugmentation de l'intensité du travail du sol améliore parfois la maîtrise des espèces concurrentes et augmente ainsi la croissance des arbres plantés (Thiffault et al. 2012). Les sylviculteurs désirent vérifier le potentiel de la préparation mécanique des sols comme méthode de rechange aux phytocides dans la maîtrise du nerprun. L'objectif de cette étude était ainsi d'évaluer l'effet de la préparation mécanique du sol sur l'envahissement de plantations d'épinette blanche par le nerprun bourdaine et de comparer l'efficacité de ces traitements à celle d'un scénario courant d'applications répétées d'un phytocide chimique (suivant un traitement de mise en andains). Notre première prédiction était que la hauteur et le recouvrement du nerprun diminueraient suivant le hersage ou le broyage du sol, au même titre que dans les parcelles traitées chimiquement, en comparaison avec la mise en andains seule. Conséquemment, notre deuxième prédiction était que la taille des plants dépinette blanche augmenterait avec les traitements de hersage ou de broyage, tout comme dans les parcelles traitées chimiquement, par rapport à la mise en andains seule. Pour vérifier ces prédictions, nous avons mesuré la hauteur et le recouvrement du nerprun ainsi que la taille des plants dépinette blanche quatre ans après leur mise en terre dans un dispositif expérimental comprenant trois traitements mécaniques du sol, incluant la mise en andains comme témoin, et un scénario d'applications répétées d'un phytocide chimique (suivant une mise en andains).

\section{Matériels et Méthodes \\ Zone d'étude}

La zone détude était localisée dans la région administrative de l'Estrie, au Québec, Canada. Cette zone se situe dans le domaine bioclimatique de lérablière à tilleul (Saucier et al. 2009; Fig. 1). La température moyenne annuelle dans cette région est de $6,6^{\circ} \mathrm{C}$ (Environnement Canada 2017). Les précipitations annuelles totales varient entre $1000 \mathrm{~mm}$ et 1150 $\mathrm{mm}$. Les degrés-jours de croissance $\left(\geq 5,0^{\circ} \mathrm{C}\right)$ varient de 1650 à 1900 et la longueur de la saison de croissance est comprise entre 165 et 190 jours (Saucier et al. 2009).

\section{Dispositif expérimental}

Le dispositif expérimental comprenait quatre traitements répétés dans quatre blocs carrés de 2.25 hectares chacun (Fig. 1). Les trois traitements de préparation mécanique du sol 


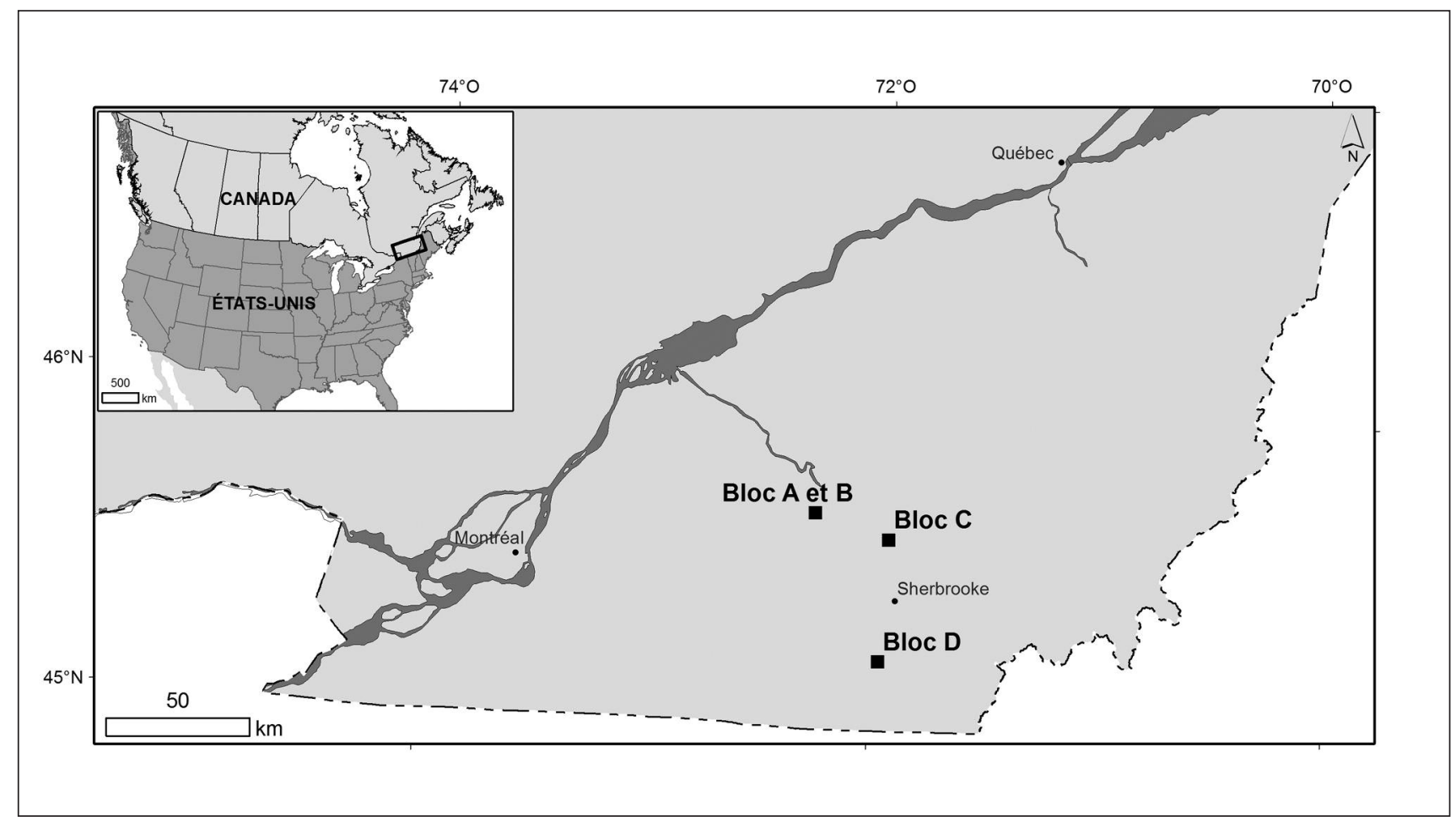

Fig. 1. Localisation des blocs du dispositif expérimental, dans la région de l'Estrie, au Québec.

étaient la mise en andains (à titre de témoin; Fig. 2), le hersage forestier et le broyage forestier (voir ci-bas; Fig. 2). Le quatrième traitement était la mise en andains suivie de l'application répétée d’un phytocide chimique (voir ci-bas; Fig. 2). Les blocs se trouvaient sur des tills minces ou épais. Tous les blocs étaient situés sur des pentes régulières variant de 2 à $10 \%$ avec des humus de type mull. Le drainage variait d'imparfait à bon, selon les blocs, lesquels ont été positionnés afin de limiter les variations de conditions au sein d'une même répétition. Les peuplements forestiers de chacun des blocs ont été récoltés en 2012 par coupe totale avec protection de la régénération et des sols. Avant la récolte, les peuplements étaient dominés par le peuplier (Populus tremuloides Michx. et P. balsamifera L.) ou le thuya (Thuja occidentalis L.), selon le site. Leur surface terrière variait de 24 à $31 \mathrm{~m}^{2} /$ ha et leur hauteur, de 17 à $18,5 \mathrm{~m}$. La densité de nerprun présent en sous-étage variait de 0,1 à 2 tiges $/ \mathrm{m}^{2}$, avec une hauteur moyenne de $3,4 \mathrm{~m}$ pour les tiges les plus hautes. Chacun des blocs a ensuite été divisé en quatre unités expérimentales carrées de 0.25 ha chacune. Les quatre traitements ont été attribués aléatoirement aux unités expérimentales de chaque bloc et ont tous été réalisés la même année que la coupe (Fig. 2). La mise en andains a été réalisée avec une pelle hydraulique équipée d'un peigne et d'un pouce. La mise en andains a peu perturbé le parterre forestier et la végétation résiduelle; ses impacts se sont limités aux effets du glissement des résidus forestiers sur le sol (Ryans et Sutherland 2001). Le hersage forestier a été réalisé à l'aide d’une herse forestière H30-24 modifiée. Tel qu’appliqué, le traitement a produit des sillons qui ont exposé le sol minéral, un mélange de matière organique et de sol minéral, et laissé une faible proportion du sol et de végétation résiduelle non perturbée. Ces parcelles comprenaient également des résidus forestiers intacts. Le broyage forestier a été effectué avec un broyeur Fecon 220 (Fecon Inc., Lebanon, Ohio). Le broyeur forestier a traité l'ensemble de la surface des parcelles, en broyant et mélangeant la végétation résiduelle, les résidus de coupe, l'humus forestier et les premiers $10-15 \mathrm{~cm}$ de sol minéral. Pour le $4^{\mathrm{e}}$ traitement, une mise en andains a été effectuée après la coupe afin de permettre l'application de 5 L/ha de glyphosate (VisionMAX, Monsanto Canada Inc,, Winnipeg, Manitoba) qui a eu lieu durant la feuillaison de juin 2013, septembre 2014, septembre 2015 et septembre 2016. À chaque fois, l’application de glyphosate a été réalisée avec un véhicule tout-terrain équipé d'un réservoir, d'une pompe et d'un tuyau permettant l'application manuelle.

Le printemps suivant l'application des traitements de préparation du terrain, nous avons reboisé les unités expérimentales avec des plants de fortes dimensions (hauteur moyenne: $43 \mathrm{~cm}$; diamètre moyen: $9 \mathrm{~mm}$ ) dépinette blanche pour obtenir une densité de 2000 plants à l'hectare. Les plants étaient issus de croisements dirigés réalisés dans le cadre du programme d’amélioration génétique des essences forestières au Québec (Beaulieu et al. 2009). Ils ont été produits à racines nues dans une pépinière gouvernementale. Dans chaque unité expérimentale, nous avons ensuite délimité une placette carrée de $400 \mathrm{~m}^{2}$ à l'intérieur de laquelle chacun des plants dépinette blanche a été identifié avec un numéro unique pour le suivi à long terme de la croissance $(\mathrm{n}=1776$ plants). Au moment de la mise en terre des plants dépinette blanche, les placettes étaient dominées par des plantes latifoliées, par le nerprun d'une hauteur modale moyenne de $30 \mathrm{~cm}$, par des arbustes non commerciaux indigènes et par des graminées. Quatre ans après la mise en terre des plants dépinette blanche, nous avons mesuré leur hauteur $(\mathrm{cm})$ et leur diamètre au niveau du sol 


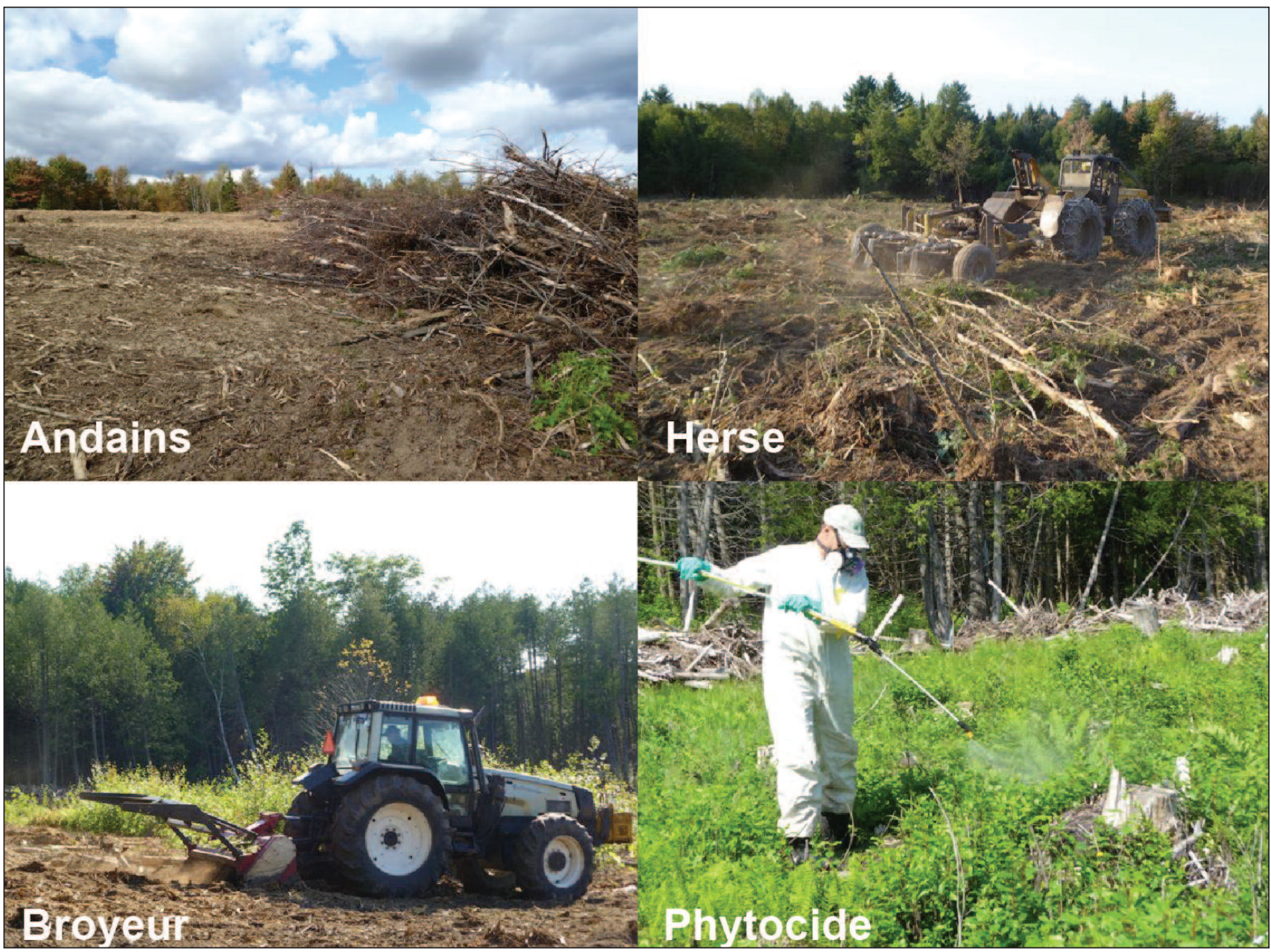

Fig. 2. Photos des traitements comparés dans cette expérience. La mise en andains a été réalisée avec une pelle hydraulique équipée d'un peigne et d'un pouce. Le hersage forestier a été réalisé à l'aide d'une herse forestière H30-24 modifiée. Le broyage forestier a été effectué avec un broyeur Fecon 220 (Fecon Inc., Lebanon, Ohio). Le traitement aux phytocides a été précédé d'une mise en andains et a consisté en l'application de 5 L/ha de glyphosate (VisionMAX, Monsanto Canada Inc,., Winnipeg, Manitoba) de manière annuelle, de 2013 à 2016 inclusivement (quatre applications).

(mm). Ensuite, nous avons estimé la hauteur modale du nerprun dans un rayon de $0,5 \mathrm{~m}$ autour des plants. Enfin, nous avons évalué le pourcentage de recouvrement du nerprun (classes de $5 \%$ ) à l'intérieur de placettes circulaires de $12,6 \mathrm{~m}^{2}$ (rayon $=2 \mathrm{~m}$ ) localisées aux coins des placettes de $400 \mathrm{~m}^{2}$.

\section{Analyses}

La hauteur et le diamètre des plants dépinette blanche ainsi que le recouvrement et la hauteur modale du nerprun ont été analysés avec des modèles linéaires généralisés mixtes. Nous avons considéré le traitement comme un effet fixe et le bloc comme un effet aléatoire. Nous avons vérifié les postulats d'homogénéité et de normalité de la variance en utilisant une approche graphique et avons fait les transformations appropriées lorsque nécessaire. Un seuil a de 0,05 a été utilisé afin d'identifier les effets significatifs. Les comparaisons entre les traitements ont été réalisées par des tests de comparaisons de moyennes a posteriori. Nous avons réalisé les analyses dans lenvironnement $\mathrm{R}$ version 3.4.1 (R Development Core Team 2017) avec la librairie nlme (Pinheiro et al. 2017).

\section{Résultats}

Quatre ans après la mise en terre des plants d'épinette blanche, les traitements ont eu un effet significatif sur l'ensemble des variables, sauf sur la hauteur des plants (Tableau 1; Fig. 3A). La hauteur des plants dépinette blanche était similaire entre les placettes traitées au broyeur forestier, avec la herse forestière ou mises en andains $(p \geq 0,992)$. Le diamètre des plants dépinette blanche (Fig. 3B) était plus élevé dans les placettes traitées de manière répétée au phytocide que dans les placettes ayant subi les autres traitements $(p \geq 0,005)$.

Le recouvrement du nerprun était plus bas dans les placettes traitées de manière répétée au phytocide que dans celles traitées au broyeur forestier $(p=0,049)$ ou avec la herse forestière ( $p=0,042)$ (Fig. 3C). Toutefois, le recouvrement du nerprun dans les placettes mises en andains était similaire au recouvrement du nerprun dans les placettes traitées de manière répétée au phytocide $(p=0,112)$. Le recouvrement du nerprun était similaire entre les placettes traitées au broyeur forestier, avec la herse forestière ou mises en andains ( $p \geq 0,898$; Fig. 3C). 
Tableau 1. Analyse de la variance de l'effet des traitements de préparation de terrain et de gestion de la végétation sur le nerprun et plants d'épinette blanche, quatre saisons de croissance après la mise en terre

\begin{tabular}{lcc}
\hline Variables & F & $\boldsymbol{p}$ \\
\hline $\begin{array}{l}\text { Nerprun bourdaine } \\
\quad \text { Recouvrement }\end{array}$ & 4,45 & 0,035 \\
$\quad$ Hauteur modale & 5,04 & 0,026 \\
Plants dépinette blanche & & \\
$\quad$ Hauteur & 0,99 & 0,442 \\
$\quad$ Diamètre & 15,03 & $<0,001$ \\
\hline
\end{tabular}

Note. Les degrés de liberté au numérateur et au dénominateur sont de 3 et 9 pour l'ensemble des variables. L'analyse des données de recouvrement a été réalisée sur les données transformées par le logarithme.
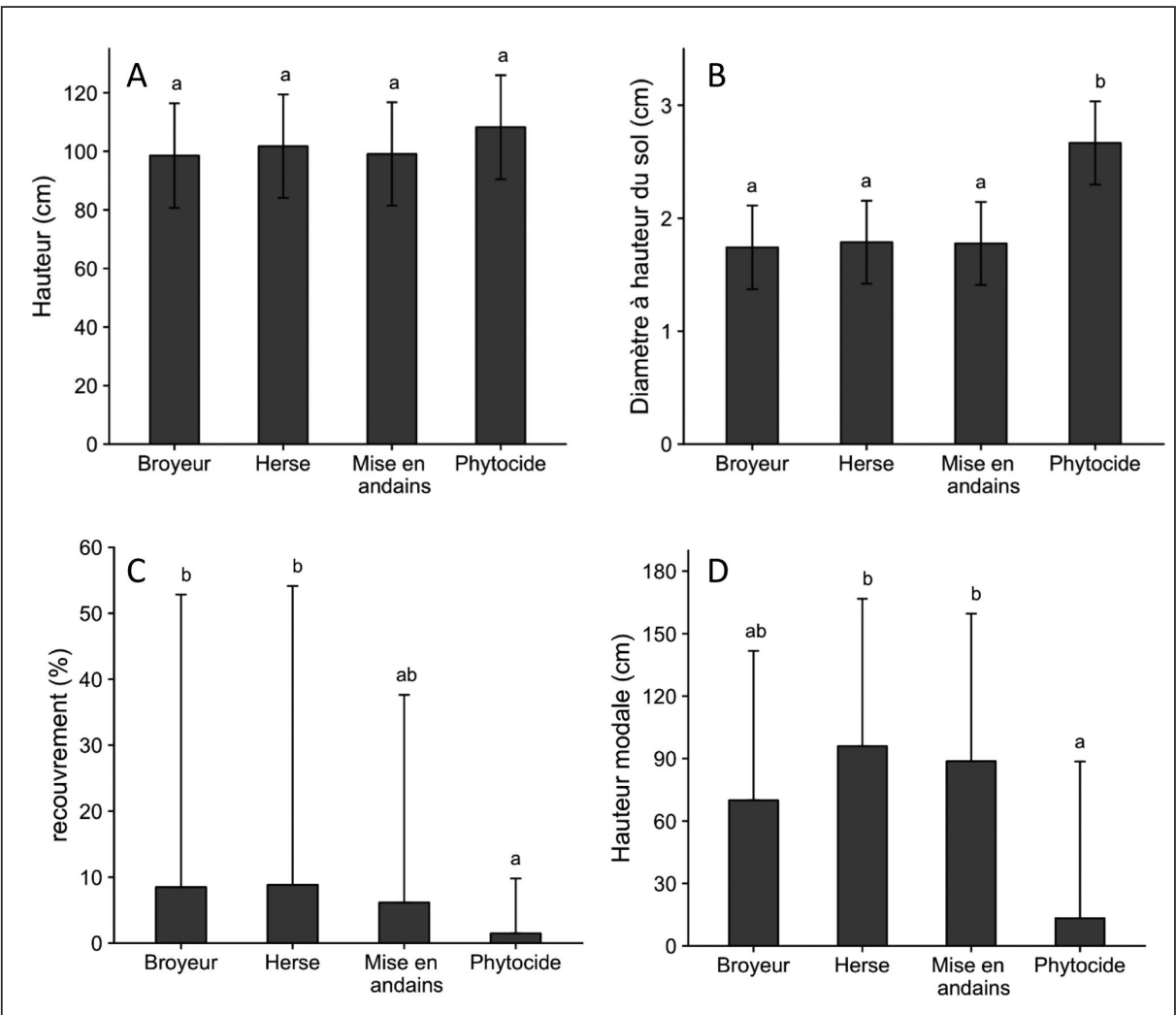

Fig. 3. Hauteur (A) et diamètre (B) des plants d'épinette blanche, ainsi que le recouvrement (C) et la hauteur modale (D) du nerprun en fonction des différents traitements, quatre saisons de croissance après la mise en terre des plants. Pour une variable donnée, les colonnes surmontées d'une même lettre ne sont pas différentes au seuil $\alpha=0,05$. Les données sont présentées sous la forme moyenne \pm IC $95 \%$. En C, les données sont celles détransformées à partir de l'échelle d'analyse (log), avec correction pour le biais.

La hauteur modale du nerprun était plus basse dans les placettes traitées de manière répétée au phytocide que dans celles traitées avec la herse forestière $(p=0,025)$ ou celles mises en andains ( $p=0,040$; Fig. 3D). Toutefois, la hauteur modale du nerprun dans les placettes traitées au broyeur forestier était similaire à la hauteur modale du nerprun dans les placettes traitées de manière répétée au phytocide $(p=$
0,142). La hauteur modale du nerprun était similaire entre les placettes traitées au broyeur forestier, avec la herse forestière ou mises en andains ( $p \geq 0,651$; Fig. 3D).

\section{Discussion}

Globalement, la préparation mécanique du sol par hersage ou broyage n'a pas eu d'effet sur l'envahissement des plantations d'épinette blanche par le nerprun bourdaine, lorsque comparée à la mise en andains seule. Ainsi, contrairement à notre première prédiction, la hauteur et le recouvrement du nerprun nont pas diminué avec ces traitements, lesquels sont plus intenses que la mise en andains seule sur la base de leurs effets sur la végétation résiduelle, l'humus et le sol minéral. Nous croyons que l'absence de différence marquée entre les traitements à l'égard de la hauteur et du recouvrement du nerprun est due aux modes de régénération efficaces de l'espèce. Alors que la mise en andains seule a possiblement permis de conserver davantage de tiges intactes de nerprun que les autres traitements, les tiges cassées par le déplacement des débris de coupe ont pu stimuler la multiplication végétative par rejets de souches (Boulet et al. 2013). Pour leur part, malgré qu'ils aient détruit la majeure partie des tiges, le hersage et le broyage peuvent avoir eu des effets similaires à ceux de la mise en andains sur la reproduction végétative, tout en favorisant la germination de nouveaux individus à partir de la banque de graines accumulée par l'espèce (Frappier et al. 2003).

Par ailleurs, la mise en andains et le broyage forestier ont eu un effet similaire à celui de l'application répétée du phytocide sur le recouvrement et la hauteur modale du nerprun, respectivement. Toutefois, seulement l'application répétée de phytocide a significativement diminué à la fois le recouvrement et la hauteur modale du nerprun par rapport aux autres traitements. Le glyphosate est un phytocide systémique transporté dans l'ensemble de la plante par les tissus vasculaires; il bloque l'action d'un enzyme essentiel dans une voie métabo-

lique (Gomes et al. 2014) et cause, le plus souvent, la mort des plantes qui l'absorbent. Ainsi, l'application répétée du phytocide a probablement tué, à chaque année, les nouveaux semis de nerprun qui émergeaient après la mort du couvert dominant de l'espèce. Après quatre années d'applications répétées du phytocide, il apparait que la réserve de graines ait commencé à diminuer suffisamment pour que l'effet devienne 
apparent à la fois sur la hauteur des tiges et le recouvrement, et ce, malgré la stimulation initiale de la reproduction végétative occasionnée par la mise en andains. Ainsi, en prenant en compte l'ensemble des variables étudiées, la préparation mécanique du sol par hersage ou broyage ne semble pas pouvoir remplacer l'application répétée d'un phytocide précédée d'une mise en andains pour limiter l'envahissement de plantations d'épinette blanche par le nerprun bourdaine. D’autres études ont aussi démontré que l'application répétée de phytocides ne pouvait pas être remplacée complètement par une méthode de rechange pour limiter l'envahissement par cette espèce (Nagel et al. 2008; Au et Tuchscherer 2014).

Contrairement à notre deuxième prédiction, la taille des plants dépinette blanche n'a pas augmenté suite au hersage ou au broyage, par rapport à la mise en andains seule. Toutefois, le diamètre des plants dépinette blanche était plus élevé après l'application répétée du phytocide (suite à une mise en andains) qu'après la seule préparation mécanique du sol. À l'instar de Lanzer et al. (2017), nous avons observé que l'application d'un phytocide chimique a eu un effet positif sur le diamètre des plants en diminuant la compétition causée par le nerprun. La croissance en diamètre est en effet très sensible à la présence de concurrents (Lanner 1985; Wagner et al. 1999; Jobidon 2000; Thiffault et al. 2014a). L'absence d'effet des traitements sur la hauteur des plants s'explique, quant à elle, par le fait que les méristèmes apicaux des conifères sont des puits (sink) puissants pour les produits de photosynthèse; ils constituent une priorité d'allocation des ressources (Lanner 1985). Ainsi, la croissance en hauteur des conifères est peu affectée par la compétition pendant les premières années suivant la mise en terre. Les bénéfices de la maîtrise de la végétation sur cette variable ne se font sentir que tardivement (Jobidon et al. 1998; Wagner et al. 1999; Thiffault et al. 2014a; Thiffault et al. 2014b).

\section{Conclusion}

Nos résultats à court terme démontrent que la préparation mécanique du sol par hersage ou broyage ne peut remplacer l'application répétée d'un phytocide chimique pour limiter l'envahissement par le nerprun des sites reboisés. L'envahissement par le nerprun soulève donc un enjeu majeur de maintien de la productivité des écosystèmes plantés, particulièrement dans un contexte où les phytocides chimiques ne peuvent être utilisés en forêt publique (Thiffault et Roy 2011). Les changements globaux risquent d'exacerber cet enjeu, de même que celui associé à l'envahissement des écosystèmes naturels en facilitant l'expansion du nerprun dans de nouvelles aires de distribution (Mack et al. 2000; Pimentel et al. 2002; Hulme 2009; Morse et al. 2014; Lennon 2015). Le développement de méthodes de rechange aux phytocides chimiques devrait se poursuivre de façon à en limiter les impacts lorsque les traitements chimiques ne sont pas accessibles.

\section{Remerciements}

Nous remercions Mario Dionne, de l'Agence de mise en valeur de la forêt privée de l'Estrie, pour sa collaboration dans la mise en place et le suivi du dispositif expérimental. Nous remercions également les partenaires régionaux pour leur contribution à létablissement de cette étude, ainsi que les propriétaires privés chez qui se sont déroulés les travaux. Nous sommes redevables à Maïé Brémont, Jacques Carignan et Évelyne Gaillard pour la qualité de leur travail technique, et aux étudiants d'été et stagiaires qui ont collaboré à la prise de mesure. Les commentaires constructifs de deux réviseurs anonymes et d'un éditeur associé ont permis d'améliorer une première version de ce manuscrit; nous les en remercions. Ce projet a été financé par l'Agence de mise en valeur de la forêt privée de l'Estrie, en collaboration avec la Direction de la recherche forestière du ministère des Forêts, de la Faune et des Parcs du Québec (projet 142332106).

\section{Références}

Au, R.C.F. et K. Tuchscherer. 2014. Efficacy of biological and chemical herbicides on non-native buckthorn during three seasonal periods. Nat. Area. J. 34: 92-98. doi: 10.3375/043.034.0110

Beaulieu, J., G. Daoust, A. Deshaies, M.S. Lamhamedi, A. Rainville et $M$. Tourigny. 2009. Amélioration génétique des arbres, gestion des vergers à graines et de semences, et production de plants forestiers. Dans Manuel de foresterie, $2^{\mathrm{e}}$ édition. Doucet, R. et M. Côté (éditeurs). Ordre des ingénieurs forestiers du Québec, Éditions Multimondes, Québec, QC.

Boulet, B., D. Marcil, J. Gobeil, I. Simard et G. Roy. 2013. Les nerpruns. Dans Le guide sylvicole du Québec, Tome 1. Les fondements biologiques de la sylviculture. Boulet, B. et M. Huot (éditeurs). Ministère des Ressources naturelles, Les Publications du Québec, Québec, QC.

Catling, P.M. et Z.S. Porebski. 1994. The history of invasion and current status of Glossy Buckthorn, Rhamnus frangula in southern Ontario. Can. Field-Nat. 108: 305-310

Converse, C.K. 1984. Element stewardship abstract for Rhamnus cathartica, Rhamnus frangula (syn. Frangula alnus). The Nature Conservancy. Arlington, VA. 14 p.

Environnement Canada. 2012. Programme de partenariat sur les espèces exotiques envahissantes. Rapport 2005-2010. Ottawa, ON. $58 \mathrm{p}$.

Environnement Canada. 2017. Données des stations pour le calcul des normales climatiques au Canada de 1981 à 2010

Fagan, M.E. et D.R. Peart. 2004. Impact of the invasive shrub glossy buckthorn (Rhamnus frangula L.) on juvenile recruitment by canopy trees. For. Ecol. Manage. 194: 95-107. doi: 10.1016/j.foreco.2004.02.015 Fiedler, A.K. et D.A. Landis. 2012. Biotic and abiotic conditions in Michigan prairie fen invaded by Glossy Buckthorn (Frangula alnus). Nat. Area. J. 32: 41-53. doi: 10.3375/043.032.0106

Frappier, B., R.T. Eckert et T.D. Lee. 2003. Potential impacts of the invasive exotic shrub Rhamnus frangula L. (glossy buckthorn) on forests of southern New Hampshire. Northeastern Naturalist 10: 277-296

Gassmann, A., I. Tosevski et L. Skinner. 2008. Use of native range surveys to determine the potential host range of arthropod herbivores for biological control of two related weed species, Rhamnus cathartica and Frangula alnus. Biol. Control. 45: 11-20. doi: 10.1016/j.biocontrol.2007.12.004

Gomes, M.P., E. Smedbol, A. Chalifour, L. Henault-Ethier, M. Labrecque, L. Lepage, M. Lucotte et P. Juneau. 2014. Alteration of plant physiology by glyphosate and its by-product aminomethylphosphonic acid: an overview. J. Exp. Bot. 65: 46914703. doi:10.1093/jxb/eru269

Haber, E. 2002. Spread and impact of alien plants across Canadian landscapes. Dans Alien Invaders in Canada's Waters, Wetlands, and Forests. Claudi, R., P. Nantel et E. Muckle-Jeffs (éditeurs). Natural Resources Canada, Canadian Forest Service, Ottawa, ON.

Hackett, R.A., H.M. Karbowski, C.M. Richards et A.K. Monfils. 2014. Status and strategy for glossy buckthorn (Frangula alnus Mill.) management. Michigan Department of Environmental Quality. Central Michigan University, Lansing, MI. 
Hamelin, C., D. Gagnon et B. Truax. 2015. Aboveground biomass of glossy buckthorn is similar in open and understory environments but architectural strategy differs. Forests. 6: 1083-1093. doi : $10.3390 /$ f6041083

Hamelin, C., B. Truax et D. Gagnon. 2016. Invasive glossy buckthorn impedes growth of red oak and sugar maple under-planted in a mature hybrid poplar plantation. New For. 47: 897-911. doi: 10.1007/s11056-016-9551-7

Hamelin, C., D. Gagnon et B. Truax. 2017. Exotic invasive shrub glossy buckthorn reduces restoration potential for native forest herbs. Sustainability 9: 1-12. doi: 10.3390/su9020249

Howell, J.A. et W.H. Jr. Blackwell. 1977. The history of Rhamnus frangula (glossy buckthorn) in the Ohio flora. Castanea 42: 111-115 Hulme, P.E. 2009. Trade, transport and trouble: Managing invasive species pathways in an era of globalization. J. Appl. Ecol. 46: 10-18. doi: 10.1111/j.1365-2664.2008.01600.x

Jobidon, R. 2000. Density-dependent effects of northern hardwood competition on selected environmental resources and young white spruce (Picea glauca) plantation growth, mineral nutrition, and stand structural development - a 5-year study. For. Ecol. Manage. 130: 77-97. doi: 10.1016/S0378-1127(99)00176-0

Jobidon, R., L. Charette et P.Y. Bernier. 1998. Initial size and competing vegetation effects on water stress and growth of Picea mariana (Mill.) BSP seedlings planted in three different environments. For. Ecol. Manage. 103: 293-305. doi: 10.1016/S0378-1127(97) 00228-4Krock, S.L. et C.E. Williams. 2002. Allelopathic potential of the alien shrub glossy buckthorn, Rhamnus frangula L: A laboratory bioassay. J. Pennsylv. Acad. Sci. 76: 17-21

Krock, S.L. et C.E. Williams. 2002. Allelopathic potential of the alien shrub glossy buckthorn, Rhamnus frangula L: A laboratory bioassay. J. Pennsylv. Acad. Sci. 76: 17-21

Krumm, F. et L. Vítková (éditeurs). 2016. Introduced Tree Species in European Forests: Opportunities and Challenges. European Forest Institute. 419 p.

Lanner, R.M. 1985. On the insensitivity of height growth to spacing. For. Ecol. Manage 13: 143-148. doi:10.1016/0378-1127(85)90030-1 Lanzer, N.B., T.D. Lee, M.J. Ducey et S.E. Eisenhaure. 2017. Sapling white pine (Pinus strobus L.) exhibits growth response following selective release from competition with glossy buckthorn (Frangula alnus P. Mill) and associated vegetation. For. Ecol. Manage. 404: 280-288. doi:10.1016/j.foreco.2017.08.049

Lee, T.D. et J.H. Thompson. 2012. Effects of logging history on invasion of eastern white pine forests by exotic glossy buckthorn (Frangula alnus P. Mill.). For. Ecol. Manage. 265: 201-210. doi:10.1016/j.foreco.2011.10.035

Lennon, M. 2015. Nature conservation in the Anthropocene: Preservation, restoration and the challenge of novel ecosystems. Plann. Theor. Pract. 16: 285-290. doi: 10.1080/14649357.2015. 1027047

Mack, R. N., D. Simberloff, W.M. Lonsdale, H. Evans, M. Clout et F.A. Bazzaz. 2000. Biotic invasions: Causes, epidemiology, global consequences, and control. Ecol. Appl. 10: 689-710. doi: 10.1890/0012-9623(2005)86[249b:IIE]2.0.CO;2

Medan, D. 1994. Reproductive biology of Frangula alnus (Rhamnaceae) in southern Spain. Pl. Syst. Evol. 173-186. doi: 10.1007/ BF00983549

Morse, N.B., P.A. Pellissier, E.N. Cianciola, R.L. Brereton, M.M. Sullivan, N.K. Shonka, T.B. Wheeler et W.H. McDowell. 2014. Novel ecosystems in the Anthropocene: A revision of the novel ecosystem concept for pragmatic applications. Ecol. Soc. 19:12. doi: 10.5751/ES-06192-190212
Nagel, L. M., R. Gregory Corace III et A.J. Storer. 2008. An experimental approach to testing the efficacy of management treatments for glossy buckthorn at Seney National Wildlife Refuge, upper Michigan. Ecol. Rest. 26: 136-142. doi: 10.3368/er.26.2.136

Pimentel, D., S. McNair, J. Janecka, J. Wightman, C. Simmonds, C. O'Connell, E. Wing, L. Russel, J. Zern, T. Aquino et T. Tsomondo. 2002. Economic and environmental costs of alien plant, animal, and microbe invasions. Dans Biological Invasions, Economic and Environmental Costs of Alien Plant, Animal, and Microbe Species. D. Pimentel (éditeur). CRC Press, Cornell University, Ithaca, NY.

Pinheiro, J., D. Bates, S. DebRoy, D. Sarkar et R Core Team. 2017. nlme: Linear and Nonlinear Mixed Effects Models. R package version 3.1-131

Ryans, M. et B. Sutherland. 2001. Site preparation - Mechanical. Dans Regenerating the Canadian Forest: Principles and Practices for Ontario. Wagner, R.G. et S.J. Colombo (éditeurs). Fitzhenry \& Whiteside, Markham, ON.

R Development Core Team. 2017. R: A language and environment for statistical computing. R Foundation for Statistical Computing, Vienna, Austria.

Saucier, J.P., A. Robitaille et P. Grondin. 2009. Cadre bioclimatique du Québec. Dans Manuel de foresterie, $2^{\mathrm{e}}$ édition. Doucet, R. et M. Côté (éditeurs). Ordre des ingénieurs forestiers du Québec, Éditions Multimondes, Québec, QC.

Thiffault, N. et V. Roy. 2011. Living without herbicides in Québec (Canada): historical context, current strategy, research and challenges in forest vegetation management. Eur. J. Forest. Res. 130: 117-133. doi: 10.1007/s10342-010-0373-4

Thiffault, N., G. Picher et I. Auger. 2012. Initial distance to Kalmia angustifolia as a predictor of planted conifer growth. New For. 43: 849-868. doi: 10.1007/s11056-012-9324-x

Thiffault, N., R. Jobidon et A.D. Munson. 2014a. Comparing large containerized and bareroot conifer stock on sites of contrasting vegetation composition in a non-herbicide scenario. New For. 45: 875-891. doi: 10.1007/s11056-014-9443-7

Thiffault, N., F. Hébert, L. Charette et R. Jobidon. 2014b. Large spruce seedling responses to the interacting effects of vegetation zone, competing vegetation dominance and year of mechanical release. Forestry 87: 153-164. doi: 10.1093/forestry/cpt048

U.S. Congress. 1993. Harmful non-indigenous species in the United States. Office of Technology Assessment, Report OTA-F-565. Government Printing Office. Washington, DC. 391 p.

Vilà, M., J.L. Espinar, M. Hejda, P.E Hulme, V. Jarosik, J.L. Maron, J. Pergl, U. Schaffner, Y. Sun et P. Pysek. 2011. Ecological impacts of invasive alien plants: A meta-analysis of their effects on species, communities and ecosystems. Ecol. Lett. 14: 702-708. doi: 10.1111/j.1461-0248.2011.01628.x

Wagner, R.G., G.H. Mohammed et T.L. Noland. 1999. Critical period of interspecific competition for northern conifers associated with herbaceous vegetation. Can. J. For. Res. 29: 890-897. doi: 10.1139/x99-055

Wiensczyk, A., K. Swift, A.E. Morneault, N. Thiffault, K. Szuba et F.W. Bell. 2011. An overview of the efficacy of vegetation management alternatives for conifer regeneration in boreal forests. For. Chron. 87: 175-200. doi: 10.5558/tfc2011-007 